\title{
Differentiated Thyroid Cancer: Management and Treatment in a Community Hospital and Guidelines to Lower Morbidity
}

\author{
Hamed H. Tewfik, MD'; Ferial A. Tewfik, MD, PhD; Thomas Viner, MD²; Dwayne Capper, MD; \\ John M. Buatti, MD**
}

'Department of Radiation Oncology, lowa City Cancer Treatment Center, lowa City, lowa, USA

2Department of Otolaryngology, Mercy lowa City Hospital, lowa City, lowa, USA

${ }^{3}$ Department of Otolaryngology, lowa City Ambulatory Surgery Center, lowa City, lowa, USA

${ }^{4}$ Department of Radiation Oncology, University of lowa Hospitals and Clinics, lowa City, lowa, USA

\begin{abstract}
Introduction: Well differentiated thyroid cancer (DTC) includes papillary and follicular cancer and has an excellent prognosis (>95\% 10-year survival). Its incidence has increased in recent decades. These tumors originate from follicular thyroid cells. For DTC, surgery and adjuvant radioiodine therapy (RIT) followed by levothyroxine administration to suppress the thyroid stimulating hormone (TSH) continues to be standard treatment.

Methods: We retrospectively reviewed a series of 119 consecutive patients treated for DTC in a community hospital by the same team of physicians from 2002 to 2016 with follow-up ranging from 2 years to 16 years. Papillary and papillary follicular subtypes account for the majority of cases. Outcomes and treatments were determined by chart review.

Results: Most patients were treated with total thyroidectomy followed by radioiodine ablation of the thyroid remnant to facilitate serologic surveillance and reduce recurrence risk. Local control was achieved in $97 \%$ of patients after initial treatment. Four patients had recurrence.

Conclusion: The risks and benefits of de-intensification strategies with decreased surgery or radioiodine dose are discussed. Strategies to individualize therapy are suggested.
\end{abstract}

\section{INTRODUCTION}

Differentiated thyroid cancer (DTC) is generally sporadic in its occurrence. DTC includes papillary and follicular cancer and has an excellent prognosis (>95\% 10-year survival) [1]. Thyroid nodules can be palpated in about $5 \%$ of women and $1 \%$ of men living in iodine sufficient areas of the world. Approximately $90 \%$ of thyroid nodules are benign. The clinical importance of thyroid nodules is to detect those harboring cancer (approximately 10\%) [2]. A diagnostic approach that uses ultrasound imaging and when indicated, fine needle aspiration (FNA) biopsy and molecular testing, facilitates individualized high-quality care and minimizes cost and unnecessary testing [2]. The diagnostic cornerstone of thyroid nodules continues to be the ultrasound examination. It should be performed in any case of known or suspected thyroid nodules or cervical lymphadenopathy to assess if further diagnostic testing is needed. FNA is the most accurate and effective method to evaluate thyroid nodules [2].

Over the last few decades, the incidence of DTC has increased worldwide [3]. It is the most common cancer in American adults aged 16-33 years [4]. Radiation is the most established cause of thyroid cancer due to the DNA damage from radiation that results in oncogenic mutation. DTC includes papillary and follicular carcinoma. DTC represents $90 \%$ of all types of thyroid cancer. Prognosis is excellent when treated according to established guidelines. For DTC standard therapy includes surgery followed by radioiodine therapy (RIT) followed by a suppressive dose of Levothyroxine. It is recommended to measure the serum thyroid stimulating hormone (TSH) level when a thyroid nodule is found. Also, a radionuclide I [1-3] single photon emission computed tomography uptake scan and neck computed tomography scan should be performed. Furthermore, the measurement of serum calcitonin is recommended to rule out medullary thyroid cancer that originates from $C$ cells and is not a differentiated thyroid cancer.
The 2015 American Thyroid Association guidelines recognize active surveillance as an alternative to immediate surgery with very low risk DTC [5]. These guidelines, however, also note that the selection of patients for surveillance as well as the definition of optimal surveillance remain poorly defined. Furthermore, the optimal dose for RIT in those receiving treatment to avoid recurrence yet minimize late toxicity also remains to be defined.

In rare cases tumors may be radioiodine-refractory and in this case the prognosis becomes poor. Identifying such patients prospectively is an area of important research. Additional options including strict suppression of TSH and external radiation therapy [6] may then be recommended. Systemic chemotherapy does not currently play a role in primary management of DTC. Recently, tyrosine kinase inhibitors have been approved for the treatment of radioiodine-refractory DTC. These drugs can slow progression but frequently are associated with significant side-effects [4].

\section{METHODS}

Records from patients seen in consultation and treated at a community hospital for differentiated thyroid cancer were retrospectively reviewed. This cohort included review of a series of 119 consecutive patients treated for DTC by the same team of physicians from 2002 to 2016 . Follow-up ranged from 2 years to 16 years. Pathology review revealed papillary and papillary follicular subtypes accounted for the majority of cases. Outcomes and treatments were determined by chart review.

\section{RESULTS}

The population consisted of 89 females and 30 males. Ages ranged from 19 to 


\begin{tabular}{|c|c|c|c|c|c|c|c|c|}
\hline \multicolumn{2}{|c|}{ Age (years) } & No. of Cases & Female & Male & Stage I & Stage II & Stage III & State IV \\
\hline \multicolumn{2}{|l|}{$11-20$} & 3 & 3 & & 3 & & & \\
\hline \multicolumn{2}{|l|}{$21-30$} & 13 & 10 & 3 & 13 & & & \\
\hline \multicolumn{2}{|l|}{$31-40$} & 17 & 11 & 6 & 17 & & & \\
\hline \multirow[t]{2}{*}{$41-50$} & $41-44$ & 13 & 12 & 1 & 13 & & & \\
\hline & $45-50$ & 13 & 9 & 4 & 10 & 1 & 1 & 1 \\
\hline \multicolumn{2}{|l|}{$51-60$} & 20 & 15 & 5 & 11 & 6 & 3 & \\
\hline \multicolumn{2}{|l|}{$61-70$} & 18 & 14 & 4 & 9 & 6 & 2 & 1 \\
\hline \multicolumn{2}{|l|}{$71-80$} & 18 & 12 & 6 & 7 & 6 & 5 & \\
\hline \multicolumn{2}{|l|}{$81-90$} & 4 & 3 & 1 & & 1 & 2 & 1 \\
\hline \multicolumn{2}{|l|}{ Total } & 119 & 89 & 30 & 83 & 20 & 13 & 3 \\
\hline
\end{tabular}

Table 2. Staging

\begin{tabular}{|ll}
\hline Stage I & Intrathyroid \\
\hline Stage II & Neck lymphadenopathy \\
\hline Stage III & Locally invasive disease \\
\hline Stage IVa & Tumor has grown extensively beyond the thyroid gland towards the spine, skeletal muscle, laryngeal nerve or large blood vessels \\
\hline Stage IVb & Distant metastases \\
\hline
\end{tabular}

88 years. Most tumors $(n=110)$ were papillary or a follicular variant of papillary thyroid cancer. Two of these were characterized as tall cell variants. Eight patients had follicular thyroid cancer and one patient had a Hürthle cell thyroid cancer. Patients were staged using the staging system as published in Swift et al. [7] shown in Table 2.

While most patients were controlled with the standard therapy outlined, a report of advanced stage and high-risk patients as well as treatment failures is informative. Three patients presented with Stage IVa disease. The first one was a male in the 45-50 years age bracket with bilateral papillary carcinoma, characterized by extensive local disease with multiple neck and upper mediastinal lymph nodes as well as lymphovascular space invasion and perineural invasion. He recurred a year after standard treatment with thyroidectomy, RIT and levothyroxine suppression treatment, declined further treatment and died. The second stage IVa patient was a 70 years old female with Hürthle cell carcinoma of the thyroid gland, treated per standard guidelines and is doing well with no evidence of disease (NED). The third stage IVa patient was an 83 years old female with bilateral papillary carcinoma, with extension into soft tissues and the anterior tracheal wall, treated with thyroidectomy, RIT and levothyroxine and is also doing well without evidence of disease recurrence.

Two patients had papillary carcinoma tall cell variants. The first patient was a 71 years old woman with stage II disease: $\mathrm{T}_{3} \mathrm{~N}_{0} \mathrm{M}_{0}$ treated with surgery, RIT and external beam radiotherapy to the thyroid bed and adjacent neck nodes, who recurred locally and was treated with a second dose of $151.1 \mathrm{mCi}$ $I^{131} 2.5$ years after her initial treatment. She is alive and doing well without recurrence. The second patient with tall cell carcinoma was an 83 years old male with Stage III disease $\mathrm{T}_{4} \mathrm{~N}_{0} \mathrm{M}_{0}$ tumor that was invading extrathyroid soft tissues and was treated with surgery and adjuvant RIT successfully and remains NED.

Four patients recurred locally after initial treatment. The first is a 70 years old male with Stage III $\left(\mathrm{T}_{4} \mathrm{~N}_{1} \mathrm{M}_{0}\right)$ papillary carcinoma, with a positive neck node that pathologically showed extracapsular extension and invasion of adjacent skeletal muscle; treated with surgery, RIT and post-operative external beam radiation therapy (EBRT) to the thyroid bed and adjacent neck nodes to a dose of 54 Gy in 27 fractions. He recurred twice locally in the neck and each time was treated with resection followed by radioactive iodine ablation $(150.1 \mathrm{mCi}$ and $250.7 \mathrm{mCi}$ respectively).

He developed lung metastases following these local recurrences and was treated with radioactive ablation using $251.0 \mathrm{mCi}$ but did not respond and the disease progressed, and he died. The second case of local recurrence was in a 71 years old female with Stage II disease $\left(\mathrm{T}_{3} \mathrm{~N}_{0} \mathrm{M}_{0}\right)$ papillary carcinoma, tall cell variant (noted previously), with capsular invasion and abundant mitotic activity. She was treated with surgery, RIT 150.8 and EBRT to the thyroid bed area and adjacent neck nodes to a dose of $54 \mathrm{~Gy}$ in 27 fractions. She recurred locally and was treated with a second dose of RIT (151.1 mCi), responded well, and is alive without evidence of disease. The third case of local recurrence was in a 45 years old male with papillary carcinoma Stage IVa $\left(\mathrm{pT}_{4} \mathrm{~N}_{1} \mathrm{M}_{0}\right)$, treated with surgery, adjuvant RIT $158.5 \mathrm{mCi}$ and post-operative EBRT to the thyroid bed and neck nodes to a dose of 54 Gy in 27 fractions. He recurred one year after treatment, declined further treatment, progressed and died from disease. The fourth case of local recurrence was in a 72 years old male with papillary Stage III carcinoma $\left(\mathrm{T}_{4 \mathrm{a}} \mathrm{N}_{1} \mathrm{M}_{0}\right)$, treated with surgery and adjuvant RIT (155.1 $\mathrm{mCi}$ ), EBRT to the thyroid bed and adjacent nodes to a dose of 54 Gy in 27 fractions. He did well for seven years following this therapy but then recurred locally and received a second dose of RIT (160.0 mCi), responded well but then recurred after two years. It was noted he discontinued his suppressive dose of levothyroxine and on his 2-year check-up visit his thyroglobulin was 274 (normal range is 2-35). He had a PET/CT neck and whole-body scan significant for metabolically positive uptake in the left supraclavicular lymph nodes which were biopsied and positive for papillary thyroid metastases. In addition, he had multiple pulmonary metastases present. He was treated with a third dose of RIT (160.5 mCi) but did not respond and died after a few months due to disease progression.

In summary, three patients died because of disease progression. All were males: one 45 years, the second 70 years and the third 72 years at the time of diagnosis. The incidence of DTC is lowest in the early teens and increases thereafter with each decade of life: it peaks in the fifth and sixth decades. Survival is better in patients diagnosed at younger age than in older patients. Generally, the dose of RIT used in this series was in the range of 150 to 170 millicuries administered orally.

\section{DISCUSSION}

There is an increased incidence of thyroid cancer worldwide [8]. Most thyroid cancers are differentiated cancers. They develop from thyroid follicular cells 
and histologic classification of DTC into papillary or follicular subtypes is important because these tumors have different patterns of metastatic spread. The papillary tumors tend to metastasize locally via lymphatics whereas the follicular tumors more frequently metastasize hematogenously to lungs and bones [9]. Nearly 8 of 10 DTC are papillary tumors. There are several types including mixed papillary follicular variant and tall cell subtype [10]. In our series $92 \%$ of cases were papillary cancers which is a higher percentage than the national norm.

Follicular cancer comprises about 2 of 10 DTC cases, also it is called follicular adenocarcinoma. Hürthle cell cancer is a rare variant and occurs in about $3 \%$ of DTC. It is also known as oxyphilic cell carcinoma. It is a more aggressive tumor with a higher rate of metastasis to lymph nodes and possibly a higher rate of recurrence [11]. Our one case of Hurthle cell carcinoma was well controlled and all 8 cases of follicular carcinoma were controlled as well.

Management of DTC is being re-evaluated since most patients are young and cured, hence motivating an effort to lower morbidity and cost. Total thyroidectomy has remained the backbone of curative intent therapy followed by RIT for ablation of the thyroid remnant. Recent questions being debated include the optimal extent of surgery and the need for administration of RIT for any residual thyroid remnant. The growth rate of DTC is very slow; thus, a prolonged follow up period is needed to evaluate any treatment paradigm changes that are suggested. Any new guidelines will benefit from more effective, less invasive, less morbid and less expensive procedures.

Certainly, known risk factors and staging should be considered carefully in formulating any changes in standard treatment considered. Risk factors to be considered are (i) size of the primary tumor (ii) TNM staging (iii) specific histology (iv) Extrathyroid spread of tumor (v) Age at time of diagnosis (above 45 years) $[7,12]$. De-intensification in the lowest risk groups appears a prudent first step.

\section{Surgical Management}

Thyroid lobectomy provides histological diagnosis and tumor removal with low risk of complications. Total thyroidectomy has increased risks of morbidity including: i) recurrent laryngeal nerve injury in about $2.5 \%$ of cases, very rarely bilateral. ii) hypocalcaemia (8.1\%). iii) hemorrhage. In patients with cytologically suspicious or malignant nodules, the 2015 America Thyroid Association guidelines recognize active surveillance as an alternative to surgery in patients with papillary microcarcinoma [4] consistent with low risk group de-intensification. Additional recommendations follow: i) Small $<1 \mathrm{~cm}$ intrathyroid cancers the recommendation now is to undergo active ultrasound surveillance without surgery if there are no signs of local invasion or lymph node metastases. ii) For papillary or follicular thyroid carcinoma $>1 \mathrm{~cm}$ and for all metastasized or microscopically invasive carcinoma irrespective of size, lobectomy to establish the diagnosis, followed by contralateral lobectomy with completion thyroidectomy to be followed with radioiodine ablative therapy [2] is recommended. iii) If lymph node metastases have been detected sonographically or intraoperatively, lymph node dissection in the affected compartment should be done to reduce the risk of local recurrence. The main argument against prophylactic lymph node dissection for all cases is the lack of evidence regarding a better outcome of the patients having the procedure without radiographically identified lymph node involvement and the higher complication rate due to more extensive surgical intervention, eg, vocal cord paralysis and parathyroid insufficiency [13].

\section{Radioiodine Therapy}

After total thyroidectomy, adjuvant RIT to ablate the thyroid remnant is administered using oral ${ }^{131}$ which facilitates serological surveillance using serum thyroglobulin and also reduces the recurrence rate [14].

RIT has been an established adjuvant therapy for more than 60 years. Eighty percent or more of total thyroidectomy patients have a small amount of residual tissue that requires ablation using RIT. It has been shown that ablative RIT decreases the rate of locoregional recurrence and mortality in patients followed more than 10 years and at intermediate or high-risk disease (Table
3). In patients with very low risk DTC a positive effect of RIT ablation on tumor recurrence is minimal and has not been demonstrated in prospective clinical trials. Regional or distant metastases are frequently only detected by elevated thyroglobulin levels after successful remnant ablation. It is important to detect local recurrence or distant metastases in an early stage so that potentially curable salvage can be delivered with minimal morbidity [15].

In radioiodine refractory tumors the prognosis is relatively poor and strict suppression of serum TSH and the addition of EBRT to a dose of 50-60 Gy has been helpful. In our series, 54 Gy in 27 fractions was used. Radioiodine ablation is indicated for (i) high risk DTC, (ii) Low risk DTC (to aide in surveillance) and in (iii) very low risk DTC only if there are risk factors such as advanced age. In addition, it is recommended when there is tumor activity detected by increasing serum level of thyroglobulin without macroscopically detected tumor by functional imaging. RIT with ${ }^{131}$ administration does have the potential for short term and long-term morbidity, hence its use should be considered carefully especially in younger age patients who present with small tumors with low risk of recurrence.

Early acute side-effects which occur after administration of RIT are rare with low doses, but increase in frequency with higher doses (>150 mCi). These include nausea because of transient gastritis, radiation sialadenitis with salivary gland swelling and change in taste with a dry mouth which generally lasts for a short time. Late side-effects include transient oligospermia, fibrosis and obstruction of the lacrimal ducts resulting in excessive tearing and a small increased risk of myelogenous leukemia and bladder cancer in patients who receive high doses of $\mathrm{I}^{131}$ [7].

Historically, the recommended dose was empirically derived (150-170 $\mathrm{mCi}$ ). Recently, there is a suggestion that lower doses of $100 \mathrm{mCi}^{131}$ or lower depending on risk factors may be adequate. Certainly, a higher dose could be used depending on the clinical situation and particularly at recurrence.

\section{Suppression Dose of Levothyroxine After ${ }^{131}$ Ablation}

This usually starts 24 hours after the RIT, the patient is placed on a suppressive dose of levothyroxine orally on a daily basis to suppress TSH and hence stimulation of the follicular cells that comprise the tumor. The dose is adjusted every six months according to the results of laboratory workup and the clinical condition of the patient. The degree of suppression is based on risk [16] and diligent follow up is important. Patients should be re-evaluated every six months including clinical examination, thyroid profile as well as serum thyroglobulin level and in some cases ultrasound imaging of the neck for local recurrence and neck lymphadenopathy in high risk cases. Positron emission tomography/computed tomography scans of the neck and whole-body imaging is indicated with rising levels of thyroglobulin and no evidence of recurrence seen by other imaging modalities.

\section{Limitations}

Our study is limited by the retrospective nature of the data collection and has several sources of potential bias. First our patients were gathered at a single community hospital and overwhelmingly had papillary carcinoma compared to most series, which is favorable. In addition, patients were treated consistently by a group of physicians with a tendency to treat with prevailing norms of thyroidectomy followed by RIT therapy and then levothyroxine suppression. Patient's with extensive lymph node involvement had the addition of external beam radiotherapy. Outcomes for control were outstanding (97\%). While outcomes were excellent, detailed evaluation of morbidity was not undertaken and the possibility that treatment could be decreased is now a prevailing trend and we agree this should be considered. Nonetheless, a high-risk population remains which should continue to be managed with standard approaches.

\begin{tabular}{ll}
\hline Table 3. TMN Staging & \\
\hline High Risk Group & $\mathrm{pT}_{3^{\prime}} \mathrm{pT}_{4^{\prime}} \mathrm{N}_{1}$ all $\mathrm{M}_{1}$ \\
\hline Low Risk Group & $\mathrm{pT}_{1 \mathrm{~b}^{\prime}} \mathrm{pT}_{2^{\prime}} \mathrm{cN}_{0^{\prime}} \mathrm{pN}_{0^{\prime}} \mathrm{cM}_{0}$ \\
\hline Very Low Risk Group & $\mathrm{pT}_{1 a^{\prime}} \mathrm{CN}_{0^{\prime}} \mathrm{pN}_{0^{\prime}} \mathrm{cM}_{0}$ \\
\hline
\end{tabular}




\section{CONCLUSION}

For DTC, total thyroidectomy and adjuvant RIT followed by a suppressive dose of levothyroxine are the established therapeutic procedures of choice. The treatment of DTC has changed from a one size fits all standard to a more individualized approach. The use of less complete surgery as well as decision to use RIT and the dose administered are to be considered carefully in the treatment of DTC. Surveillance for very low risk DTC is an acceptable option. The aim to lower morbidity, lower the cost of treatment and improve patient quality of life is attainable using these principles.

\section{ARTICLE INFORMATION}

*Correspondence: John M. Buatti, MD. Department of Radiation Oncology, University of lowa Hospitals and Clinics. 200 Hawkins Drive, lowa City, IA 52242, USA. E-mail: john-buatti@uiowa.edu

Received: Nov. 25, 2019; Accepted: Feb. 03, 2020; Published: Apr. 16, 2020

DOI: $10.24983 /$ scitemed.aohns.2020.00126

Ethics Approval and Consent to Participate: The study is in accordance with the ethical standards of the 1964 Helsinki declaration and its later amendments or comparable ethical standards.

Funding: The study did not receive any specific grant from funding agencies in the public, commercial, or not-for-profit sectors.

Conflict of Interest: The authors report no financial or other conflict of interest relevant to this article, which is the intellectual property of the authors.

Copyright ( $) 2020$ The Authors. This is an open-access article distributed under the terms of the Creative Commons Attribution 4.0 International License (CC-BY).

\section{REFERENCES}

1. Andresen NS, Buatti JM, Tewfik HH, Pagedar NA, Anderson CM, Watkins JM. Radioiodine ablation following thyroidectomy for differentiated thyroid cancer: Literature review of utility, dose, and toxicity. Eur Thyroid J 2017;6(4):187-196.

2. Durante C, Grani G, Lamartina L, Filetti S, Mandel SJ, Cooper DS. The diagnosis and management of thyroid nodules: A review. JAMA 2018;319(9):914-924.

3. Schmidbauer B, Menhart K, Hellwig D, Grosse J. Differentiated thyroid cancer-treatment: State of the art. Int J Mol Sci 2017;18(6)

4. Brito JP, Moon JH, Zeuren R, et al. Thyroid cancer treatment choice: A pilot study of a tool to facilitate conversations with patients with papillary microcarcinomas considering treatment options. Thyroid 2018;28(10):1325-1331.

5. Haugen BR, Alexander EK, Bible KC, et al. 2015 American thyroid association management guidelines for adult patients with thyroid nodules and differentiated thyroid cancer: The american thyroid association guidelines task force on thyroid nodules and differentiated thyroid cancer. Thyroid 2016;26(1):1-133.

6. Rooney $\mathrm{KP}$, Miah $\mathrm{AB}$, Bhide $\mathrm{SA}$, et al. Intensity modulated radiotherapy in locally advanced thyroid cancer: Outcomes of a sequential phase I dose-escalation study. Radiother Oncol 2018;127(1):43-48

7. Swift PS, Larson S, Price DC. Conacer of the Thyroid. In: Leibel SA, Phillips TL, eds. Textbook of radiation oncology. 2nd ed. Philadelpha: Saunders, 2004. 757-778.

8. Hershman JM. The increased incidence of thyroid cancer is worldwide. Clinical Thyroidology 2017;29:11-12

9. Ito Y, Miyauchi A. Prognostic factors and therapeutic strategies for differentiated carcinomas of the thyroid. Endocr J 2009;56(2):177-192.

10. Wenig BM, Thompson LD, Adair CF, Shmookler B, Heffess CS. Thyroid papillary carcinoma of columnar cell type: A clinicopathologic study of 16 cases. Cancer 1998;82(4):740753.

11. Bhattacharyya N. Survival and prognosis in hurthle cell carcinoma of the thyroid gland. Arch Otolaryngol Head Neck Surg 2003;129(2):207-210.

12. Orr LE, Yeh MW, Livhi MJ. Male sex is associated with increased mortality from papillary thyroid cancers with BRAF V600E mutation. Clinical Thyroidology 2018;30:450-452.

13. Gimm O, Drabel H, Holzheimer RG, MannickJA. Surgical treatment: evidence-based and problem-oriented. In: Holzheimer RG, Mannick JA, eds. Munich: Zuckschwerdt; 2001.

14. Ruel E, Thomas S, Dinan M, Perkins JM, Roman SA, Sosa JA. Adjuvant radioactive iodine therapy is associated with improved survival for patients with intermediate-risk papillary thyroid cancer.J Clin Endocrinol Metab 2015;100(4):1529-1536.

15. Sawka AM, Brierley JD, Tsang RW, et al. An updated systematic review and commentary examining the effectiveness of radioactive iodine remnant ablation in well-differentiated thyroid cancer. Endocrinol Metab Clin North Am 2008;37(2):457-480.

16. Do Cao C, Wemeau JL. Risk-benefit ratio for TSH-suppressive levothyroxine therapy in differentiated thyroid cancer. Ann Endocrinol (Paris) 2015;76(1 Suppl 1):1S47-52. 